\title{
Epidemiologia do câncer de mama
}

\author{
Epidemiology of breast cancer
}

O câncer de mama considerado esporádico, ou seja, sem associação com o fator hereditário, representa mais de $90 \%$ dos casos de câncer de mama em todo mundo. Dados clínicos, epidemiológicos e experimentais têm demonstrado que o risco de desenvolvimento de câncer de mama esporádico está fortemente relacionado à produção de esteroides sexuais. Condições endócrinas moduladas pela função ovariana, como a menarca precoce, menopausa tardia e gestação, assim como a utilização de estrógenos exógenos, são componentes relevantes do risco de desenvolvimento do câncer de mama ${ }^{1-3}$. Em sinergismo com os fatores hormonais, estudos observacionais indicam que o comportamento humano relacionado ao estilo de vida, o que inclui modificações na dieta e na atividade física, podem contribuir para o aumento da incidência do câncer de mama em todo o mundo ${ }^{4}$.

Segundo os dados do programa Surveillance, Epidemiology, and End Results (SEER), do Instituto Nacional do Câncer nos Estados Unidos, durante os anos de 1990 a 2000, a taxa de incidência de câncer de mama vinha aumentando cerca de $3 \%$ ao ano. $\mathrm{O}$ aumento da incidência nesse período foi atribuído, em parte, à melhora e divulgação dos programas de rastreamento do câncer de mama ${ }^{5}$. No entanto, a partir de 2003, a incidência da doença vem sofrendo um leve declínio. Dois fatores são descritos como responsáveis por essa observação. A redução da incidência em todos os grupos de mulheres com mais de 45 anos sugere um efeito devido à saturação do sistema de rastreamento da doença. Por outro lado, a redução súbita da incidência entre os anos de 2002 e 2003, que ocorreu predominantemente no grupo de mulheres entre 50 e 69 anos de idade, deve ser reflexo da redução do uso indiscriminado da terapia de reposição hormonal ${ }^{6,7}$.

Martins et al. ${ }^{8}$ analisaram o perfil epidemiológico do câncer de mama na população da cidade de Goiânia (GO) e relataram um aumento do número de casos de câncer de mama na ordem de 249\% no quinquênio 1999-2003 quando comparado ao quinquênio 1989-1993. Os autores enfatizaram que esse aumento foi decorrente da melhora no programa de rastreamento do câncer de mama na população daquela cidade.

Algumas considerações a respeito das características da população brasileira devem ser discutidas. A população rural no estado de Goiás ocupava 23,7\% da população total em 1991 e caiu para 12,1\% no ano de 2001. A população da cidade de Goiânia cresceu 22,4\% das Clínicas da Faculdade de Medicina de Ribeirão Preto da Universidade de São Paulo

Avenida dos Bandeirantes, 3.900 - Monte Alegre $14048-900$ - Ribeirão Preto (SP), Brasil E-mail: dtiezzi@usp.br Fone: 551636022488
' Professor do Setor de Mastologia e Oncologia Ginecológica do Departamento de Ginecologia e Obstetrícia da Faculdade de Medicina de Ribeirão Preto da Universidade de São Paulo - USP - São Paulo (SP), Brasil. 
entre os anos de 1991 e 2003 - um aumento total de 207.052 habitantes no período. O índice de desenvolvimento humano também aumentou de 0,778 para 0,832 nesse período para a população de Goiânia, com redução da taxa de mortalidade infantil (de 27,32, em 1990, para 20,3 por 100.000 nascidos vivos, em 2000). A arrecadação de Impostos sobre Circulação de Mercadorias e Prestação de Serviços (ICMS) no município cresceu 97,3\% somente no período que compreende os anos de 1998 e $2003^{9}$.

Estudos recentes demonstram que a urbanização da sociedade e as condições socioeconômicas são fatores consideráveis de risco para o câncer de mama ${ }^{10-12}$. Segundo Fan et al..$^{10}$, a incidência de câncer de mama aumentou de 20,16 para 71,46 casos por 100.000 mulheres, entre os anos de 1975 e 2003, em Xangai, China. Durante as três últimas décadas, o produto interno bruto (PIB) de Xangai cresceu de 27,3 bilhões, em 1978, para 1,036 trilhões, em 2006. A taxa total de fertilidade caiu de 6,4, em 1955, para 0,81, em 2006, na mesma população. Adicionalmente, a atividade física vem diminuindo na população urbana em toda a China, resultando em aumento na prevalência de obesidade ${ }^{13,14}$.

A modificação da dieta também tem sido relatada como um fator de risco. O único estudo em grande escala que demonstrou o impacto da modificação da dieta e no risco de câncer de mama foi o Women's Health Initiative Dietary Modification Trial ${ }^{15}$. O risco de desenvolvimento de câncer de mama é reduzido em mulheres que perdem peso na pré e pós-menopausa. Segundo os dados do Iowa Women's Health Study ${ }^{16}$, mulheres que perdem $5 \%$ ou mais de seu peso antes e após a menopausa apresentam uma redução do risco de desenvolver o câncer de mama na pós-menopausa na ordem de 40 e $25 \%$, respectivamente. O Nurses Health Study (NHS) demonstrou que mulheres que perdem pelo menos $10 \mathrm{~kg}$ após a menopausa têm uma redução de risco de câncer de mama na ordem de $56 \%{ }^{17}$. Tanto o aumento populacional quanto a migração da população rural para as grandes cidades, resultando em alterações significativas do estilo de vida, são fatores que devem ser levados em consideração na análise dos resultados descritos na população de Goiânia.

De fato, os resultados encontrados por Martins et al. ${ }^{8}$ são esperados. Os programas de rastreamento do câncer de mama foram divulgados mais tardiamente em nosso país se comparado com os países desenvolvidos. As mudanças no perfil da população brasileira, no que diz respeito aos estilos de vida e ao comportamento reprodutivo, são favoráveis para aumento do risco da doença. O mesmo foi observado em outros países em desenvolvimento. No entanto, devemos analisar com cautela o aumento no número de notificações de pacientes com câncer de mama relatado pelos autores. Deve ficar claro que o aumento de $249 \%$ no registro de câncer não reflete um aumento da mesma proporção na taxa de incidência da doença. Tanto os aspectos demográficos aqui discutidos como a melhoria no sistema de informação, no sistema de regulação de pacientes para tratamento em níveis terciários bem como o crescimento populacional são fatores que, certamente, interferem no resultado final da análise de dados em base populacional.

\section{Referências}

1. Greenlee RT, Murray T, Bolden S, Wingo PA. Cancer statistics, 2000. CA Cancer J Clin. 2000;50(1):7-33.

2. Lambe M, Hsieh CC, Chan HW, Ekbom A, Trichopoulos D, Adami HO. Parity, age at first and last birth, and risk of breast cancer: a populationbased study in Sweden. Breast Cancer Res Treat. 1996;38(3):305-1 1.

3. MacMahon B, Cole P, Lin TM, Lowe CR, Mirra AP, Ravnihar B, et al. Age at first birth and breast cancer risk. Bull World Health Organ. 1970;43(2):209-21.

4. Bray F, McCarron P, Parkin DM. The changing global patterns of female breast cancer incidence and mortality. Breast Cancer Res. 2004;6(6): 229-39.

5. Jemal A, Siegel R, Ward E, Hao Y, Xu J, Murray T, et al. Cancer statistics, 2008. CA Cancer J Clin. 2008;58(2):71-96.

6. Jemal A, Ward E, Thun M. Recent trends in breast cancer incidence rates by age and tumor characteristics among U.S. women. Breast Cancer Res. 2007;9(3):R28.

7. Brewster DH, Sharpe KH, Clark DI, Collins J. Declining breast cancer incidence and decreased HRT use. Lancet. 2009;373(9662):459-60.

8. Martins E, Freitas-Junior R, Curado MP, Freitas NMA, Oliveira JC, Bandeira e Silva CM. Evolução temporal dos estádios do câncer de mama ao diagnóstico em um registro de base populacional no Brasil Central. Rev Bras Ginecol Obstet. 2009;31 (5):219-23.

9. SEPIN - Superintendência de Estatística Pesquisa e Informação [Internet]. Estatísticas municipais: Goiânia. Goiânia; 2009 [citado 2009 mar 31]. Disponível em: http://portalsepin.seplan.go.gov.br/

10. Fan L, Zheng Y, Yu KD, Liu GY, Wu J, Lu JS, et al. Breast cancer in a transitional society over 18 years: trends and present status in Shanghai, China. Breast Cancer Res Treat. In press 2009. 
11. Torio CM, Klassen AC, Curriero FC, Caballero B, Helzlsover K. The modifying effect of social class on the relationship between body mass index and breast cancer incidence. Am J Public Health. In press 2009.

12. Wong IO, Cowling BJ, Schooling CM, Leung GM. Age-period-cohort projections of breast cancer incidence in a rapidly transitioning Chinese population. Int J Cancer. 2007; 121 (7):1556-63.

13. Li Y, Schouten EG, Hu X, Cui Z, Luan D, Ma G. Obesity prevalence and time trend among youngsters in China, 1982-2002. Asia Pac J Clin Nutr. 2008;17(1):131-7.

14. Bell AC, Ge K, Popkin BM. The road to obesity or the path to prevention: motorized transportation and obesity in China. Obes Res. 2002; 10(4):277-83.

15. Prentice RL, Caan B, Chlebowski RT, Patterson R, Kuller LH, Ockene JK, et al. Low-fat dietary pattern and risk of invasive breast cancer: the Women's Health Initiative Randomized Controlled Dietary Modification Trial. JAMA. 2006;295(6):629-42.

16. Harvie M, Howell A, Vierkant RA, Kumar N, Cerhan JR, Kelemen LE, et al. Association of gain and loss of weight before and after menopause with risk of postmenopausal breast cancer in the lowa women's health study. Cancer Epidemiol Biomarkers Prev. 2005; 14(3):656-61.

17. Eliassen AH, Colditz GA, Rosner B, Willett WC, Hankinson SE. Adult weight change and risk of postmenopausal breast cancer. JAMA. $2006 ; 296(2): 193-201$. 